\title{
Tracer le monde : outils et instruments de la Renaissance aux Lumières
}

Trace the World: Tools and Instruments from the Renaissance to the

Enlightenment

\section{Audrey Millet}

\section{(2) OpenEdition}

\section{Journals}

Édition électronique

URL : http://journals.openedition.org/artefact/443

DOI : $10.4000 /$ artefact. 443

ISSN : 2606-9245

Éditeur :

Association Artefact. Techniques histoire et sciences humaines, Presses universitaires du Midi

Édition imprimée

Date de publication : 1 octobre 2016

Pagination : 215-231

ISBN : 978-2-7535-5174-9

ISSN : 2273-0753

\section{Référence électronique}

Audrey Millet, "Tracer le monde : outils et instruments de la Renaissance aux Lumières », Artefact [En ligne], 4 | 2016, mis en ligne le 07 juillet 2017, consulté le 19 avril 2019. URL : http:// journals.openedition.org/artefact/443; DOI : 10.4000/artefact.443 


\section{Tracer le monde : outils et instruments de la Renaissance aux Lumières}

Audrey MILLET

\section{Résumé}

$\mathrm{Du} \mathrm{XVI}{ }^{\mathrm{e}}$ au $\mathrm{xVIII}{ }^{\mathrm{e}}$ siècle, les scientifiques, les techniciens et les pouvoirs veulent mesurer et représenter le monde plus précisément. Cette mise en ordre passe par l'amélioration des instruments de transcription. Les ingénieurs, astronomes, mathématiciens ont en commun le dessin et les instruments. Plumes à dessiner, compas et équerres sont constamment améliorés. Derrière le caractère pratique et technique des instruments, se cache l'ambition des élites. Elles cherchent à rayonner au sein d'une société convertie aux innovations. Elles montrent aussi leur foi en l'universalité des connaissances.

Mots-clefs : fabricant, instruments, livre, sciences, techniques.

\section{Abstract. Trace the world : tools and instruments from the Renaissance to the Enlightenment}

From $16^{\text {th }}$ to $18^{\text {th }}$ centurie, scientists, technicians and authorities want to measure and represent the world, more precisely. This happens by ordering the improvement transcription instruments. Engineers, astronomers, mathematicians have in Common the drawing and instruments. Pen drawing, compass and brackets are constantly improved. Behind the practical and technical character of instruments, hides the ambition of elites. They try to shine within a society converted to the innovations. They also show their faith in the universality of knowledge.

Keywords : book, instruments, maker, science, technology.

*. Audrey Millet est docteure en histoire (Paris 8) et docteure ès Lettres (Neuchâtel). Son doctorat porte sur les dessinateurs de fabrique de 1750 à 1850. Elle est actuellement Max Weber Fellow à l'European University Institute à Florence. Elle a publié des articles sur le savoir-faire, les dessinateurs et l'apprentissage. Ses travaux portent désormais sur la propriété industrielle des dessins de fabrique et sur la mode entendue comme histoire totale. Elle prépare l'édition commentée d'un journal de dessinateur (1794-1862). Contact : [audrey-patrizia@yahoo.fr.]. 
Deux siècles séparent les dessins de compas de Léonard de Vinci (14521519) de l'engouement de Samuel Pepys (1633-1703) pour le pantographe. " Il me le faut ", écrit Pepys dans son journal ${ }^{1}$. À la veille de la Révolution française, cet appareil visant à réduire ou agrandir des dessins a été maintes fois amélioré. En trois siècles, les artisans, les fabricants, les savants et les élites des cours européennes ont montré un vif intérêt pour l'amélioration et le progrès des sciences et des techniques. Néanmoins, pour matérialiser une idée, une machine, un dessin ou un procédé, il faut avoir à disposition les outils adéquats. Quelle que soit la finalité envisagée par les acteurs, tous ont compris que la capacité à reproduire est un moyen essentiel de la compréhension du monde. Au centre de ce dialogue, entre la mesure et l'idée matérialisée sous la forme d'un dessin, se situent les instruments de dessin utilisés pour la navigation, la cartographie, l'architecture, l'ingénierie militaire ou encore la décoration des textiles.

Les chercheurs se sont particulièrement intéressés aux techniques de représentation du monde ${ }^{2}$. Les traités d'architecture et les questions de perspective ont fait l'objet d'études poussées $^{3}$. Maurice Daumas et Bertrand Gille ont publié des essais précurseurs prenant en compte les objets médiateurs que sont les instruments ${ }^{4}$. Les sociologues proposent une réflexion novatrice en se rapprochant des habitudes, des gestes de l'artisan, en résumé de la "manière de faire ${ }^{5} »$. Ces vingt dernières années, les historiens ont développé une approche complexe au carrefour des techniques, de l'art, de l'économie et du social ${ }^{6}$.
Établir une typologie complète des instruments est impossible dans le cadre de cet article. Je m'intéresserai donc à des catégories d'outils largement partagées par les praticiens, notamment, les stylets, compas, règles à échelles, instruments de copie, d'agrandissement et de réduction. Afin d'entretenir la tension entre pratique, représentation et acquisition de l'instrument, je m'appuierai sur une analyse des instruments conservés dans les musées européens, sans pour autant oublier que les outils qui nous sont parvenus sont souvent les plus beaux et les plus onéreux. Il est difficile d'approcher le stylet de l'ingénieur du xvII ${ }^{\mathrm{e}}$ siècle. Les sources littéraires sont plus nombreuses. En effet, depuis le $\mathrm{xvI}^{\mathrm{e}}$ siècle, ont paru de nombreux ouvrages d'artisans ou de lettrés visant à faire un état de l'art des instruments disponibles. Des traités d'architecture ou d'ingénierie sont également disponibles. Enfin, les dessins des techniciens permettent de confronter les instruments utilisés aux recommandations théoriques des ouvrages. À partir de trois siècles d'instruments, je propose d'interroger les transformations des sciences et des techniques en lien avec une nouvelle vision du monde ${ }^{7}$. La mise en place de la mesure du monde par les techniciens de l'époque moderne tend à réconcilier le travail manuel et l'économie des savoirs.

Du xve siècle au XviII ${ }^{\mathrm{e}}$ siècle, de quelles manières les instruments du dessin participent-ils à modifier et/ou fixer les savoirs? Comment accompagnentils une lente et continue transformation de l'environnement scientifique et technique?

Je traiterai tout d'abord l'intérêt renouvelé pour les instruments de dessin 
durant les $\mathrm{Xv}^{\mathrm{e}}$ et $\mathrm{XvI}^{\mathrm{e}}$ siècles. Le Grand Siècle se caractérise par une dynamique de rationalisation des savoirs marquée par des améliorations constantes et une attention croissante des pouvoirs qui fera l'objet d'un deuxième point. Enfin, j'étudierai l'élargissement des gammes durant le $\mathrm{xVIII}^{\mathrm{e}}$ siècle et les tentatives d'universalisme des instruments portent les marques de la globalisation des savoirs.

\section{Des instruments pour décrire le monde $\left(x v^{e}-x v l^{e}\right.$ siècles)}

Caractérisé par un regain d'intérêt pour l'Antiquité gréco-romaine, l'horizon scientifique et technique des praticiens de la Renaissance s'agrandit ${ }^{8}$. Toutefois, les hommes des $\mathrm{xv}^{\mathrm{e}}$ et $\mathrm{XVI}^{\mathrm{e}}$ siècles sont résolument tournés vers l'avenir. Le développement des mathématiques induit alors l'amélioration des systèmes de mesure par les astronomes, les navigateurs, les ingénieurs militaires et les topographes ${ }^{9}$. La mise en place des règles de la perspective géométrique fait figure d'apport majeur ${ }^{10}$. Durant le $\mathrm{xv}^{\mathrm{e}}$ siècle, les instruments de dessin ont déjà fait l'objet de modifications importantes, en particulier en Italie ${ }^{11}$. Nouveaux ou améliorés, ils servent le travail $\mathrm{du}$ dessinateur qui se fournit, comme le mathématicien, au sein de l'échoppe. Il s'agit bien d'instruments transverses.

\section{Fabricant d'instruments : de Nuremberg aux centres européens}

Les principes de Vitruve correspondent aux ambitions des architectes du $x v^{e}$ siècle qui adoptent le dessin orthogonal ${ }^{12}$. Les instruments ne changent pas du tout au tout, mais les praticiens profitent des améliorations en cours dans l'horlogerie ou l'armurerie et de l'introduction progressive de l'imprimerie ${ }^{13}$. La situation géographique des villes allemandes, au carrefour du nord et du sud de l'Europe, et l'accès facilité à l'étain et au cuivre, minerais locaux, autorisent le développement de centres de fabrication. Un nouvel artisanat naît. Le métier de fabricant de compas, non corporé, figure dans le Livre des métiers de Jost Amman dès 1568 (figure 1). Un siècle plus tard, Paris, Milan et Londres ont attiré une main-d'œuvre compétente ${ }^{14}$. À Paris, les fabricants de compas sont rattachés à la corporation des couteliers et fondeurs de métaux, tandis qu'à Milan, ils s'intègrent aux armuriers ${ }^{15}$. Dans le cadre de l'atelier-échoppe se met en place un savoir spécifique visant à améliorer la précision des instruments et à faciliter le tracé. L'instrument devient le meilleur médiateur entre le monde et sa représentation repoussant les limites des possibles et des pratiques. Le premier fabricant d'instruments londonien connu est Augustine Ryther (mort en 1593). Graveur de métier, il améliore et fabrique ses propres instruments pour 


\section{Der Scircedfectimiot.}

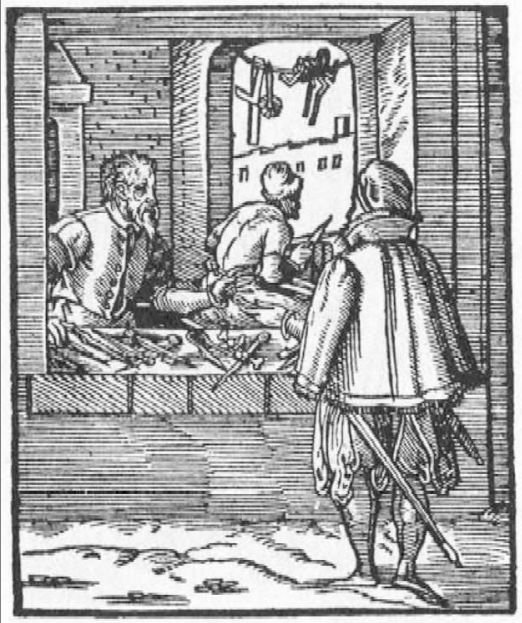

Ych mach mancherlen VBercl"şeug art/ Subtile Zircel und Dafiart/

Dancherlen Bangen / afchrauffe vĩ glate/

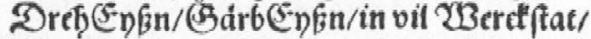

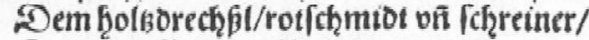
Rantolgieffer bHo Balbieret/

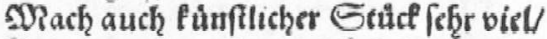
Rein gegraben/glatt vno fubtil. $x$ if

FIG. 1. - Fabricant de compas dans sa boutique. Jost Amman, Le livre des métiers ou Eygentliche Beschreibung aller Stände auff Erden, hoher und nidriger, geistlicher und weltlicher, aller Künsten, Handwercken und Händeln Durch d. weitberümpten Hans Sachsen gantz fleissig beschrieben $u$. in teutsche Reimen gefasset, Frankfurt am Mayn: Feyerabend, 1568, p. 67. réaliser des cartes plus précises ${ }^{16}$. En France, vers 1597, Philippe Danfrie (v. 1534-1606), fils d'un graveur sur métal, modifie son trigonomètre en outil de triangulation, appelé graphomètre ${ }^{17}$. Monté sur rotule, l'instrument autorise à prendre des angles entre objets situés dans un même plan. Danfrie, proche $\mathrm{du}$ pouvoir central, succède à son père comme graveur général des Monnaies en $1582^{18}$. Les gens de métier, liés ou non à $l^{\prime}$ État, sont les premiers à perfectionner leurs instruments afin de faciliter leur travail et $\mathrm{d}^{\prime}$ améliorer leurs ouvrages ${ }^{19}$. Les mécènes européens se passionnent alors pour les innovations.

\section{Le mécénat des cours européennes}

L'intérêt des Grands pour la recherche scientifique résonne largement avec leur volonté de rayonnement. À Prague, l'empereur Rodolphe II soutient le fabricant Érasme Habermel (1538-1606), dont les instruments luxueux sont offerts comme cadeaux à des collectionneurs ${ }^{20}$. Cent cinquante instruments de l'atelier d'Habermel sont inventoriés. Une quarantaine d'entre eux est signée. Les noms gravés de Francisco de Padoanis, médecin italien, Hermann Bulder, allemand, et Tycho Brahé, astronome danois, traduisent la diversité des origines des commanditaires et montrent l'engouement européen pour les instruments de mesure et de tracé. Les outils sont variés : cercles, sphères armillaires, 
cadrans solaires, astrolabes, quadrants, nécessaires de dessin, théodolites ${ }^{21}$. Les États italiens soutiennent également la production d'instruments de géométrie complexes. Les faiseurs d'instruments, métallurgistes ou orfèvres, travaillent autour des Sforza à Milan, puis des Médicis. Vers les années 1570, Cosme de Médicis est le mécène de Baldassarre Lanci. À la fois fabricant et ingénieur militaire du gonfalonier, Lanci invente un compas de triangulation pour les relevés topographiques ${ }^{22}$. En Angleterre, Thomas More (1478-1535), chancelier d'Henri VIII, invite à Hampton Court le mathématicien, astronome, horloger et fabricant allemand Nicolas Katzer (14971543). More veut faire progresser les connaissances anglaises en astronomie et en mathématiques. Quant aux premiers traités techniques avec illustrations, ils apparaissent dans la seconde moitié du $x I^{\mathrm{e}}$ siècle.

\section{Theatrum instrumentorum : entre imaginaire et pratique ${ }^{23}$}

Jacques Besson (1530-1572), mathématicien et ingénieur, rédige vers 1570 un livre de machines, dédié à Charles IX. Jacques Androuet du Cerceau et Besson publient le livre premier de Theatrum instrumentorum en 1571-1572²4. Il présente pour la première fois des machines de divers usages en séries. La réédition de 1579 contient soixante planches d'instruments. Certaines règles, des compas et des rapporteurs n'ont jamais été réalisés, relevant plus de l'imaginaire que de la pratique. L'effort de publication d'instruments, de diffusion des connaissances scientifiques et techniques, concerne aussi l'Allemagne. Le Perspectiva de
Hans Lencker (1523-1585), publié à Nuremberg en 1579, montre l'utilisation en géométrie du compas à pointes sèches ${ }^{25}$. Réédité à de nombreuses reprises, l'ouvrage insiste sur l'importance des instruments pour appuyer les savoirs mathématiques et géométriques à partir des instruments.

Les praticiens et les savants cherchent à mesurer le monde plus précisément. La compréhension de l'univers et la description rationnelle se situent au centre de leurs intérêts. La publicisation de l'information, grâce au développement de l'imprimerie, tend à mettre en commun le savoir scientifique. Astronomes, orfèvres et ingénieurs pratiquent le savoir transverse du dessin. Connaître les instruments de dessin permet ensuite de pratiquer, de comprendre et de décrire. Ces outils deviennent alors des médiateurs du monde. La pédagogie des ouvrages, construits sous la forme de traités où les instruments sont accompagnés de légendes, en fait des manuels avant l'heure. Toutefois, cet effort de publication de l'innovation et de présentation des possibles dit peu sur les réalités et l'utilisation des instruments par les praticiens. Il s'agit donc d'observer les artefacts qui nous sont parvenus.

Les stylets, instruments servant à tracer, ne sont pas une invention de la Renaissance, mais la période est marquée par une transformation majeure : les tire-lignes ne nécessitent plus de second passage car l'encre, à base de seiche ou de noix de galle et de peroxyde de fer, est utilisée directement ${ }^{26}$. L'épaisseur du trait est ajustable grâce à un anneau coulissant. Enfin, la pointe pour plume à pointiller avec système de roulette intégré est une des premières 
plumes pour spécialistes ${ }^{27}$. Le crayon subit deux transformations majeures. L'exploitation du graphite, forme cristallisée du carbone, débute dans le comté de Cumberland dans le nord-ouest de $l^{\prime}$ Angleterre à partir de $1540^{28}$. Tracer est désormais plus simple : l'épaisseur du trait est modifiable, un seul geste suffit et le graphite permet de nouveaux effets. Le compas retient particulièrement l'attention des fabricants.

Le compas ordinaire, utilisé par les charpentiers, architectes, menuisiers ou mathématiciens, est modifiéà Augsbourg et à Nuremberg ${ }^{29}$. Le développement de l'artisanat lié aux métaux, horlogerie ou bijouterie, joue un rôle dans la recherche de précision. Il est désormais possible de régler l'ouverture des branches du compas disponible en différentes tailles, s'adaptant ainsi à la taille des supports travaillés. Aussi, le compas à dessin fait son apparition : il est muni d'un emplacement pour le crayon ou pour une pointe à encre ${ }^{30}$. Dans leurs ouvrages respectifs, Pomodoro et Bion destinent ces compas à un usage militaire ou maritime $^{31}$. Vers 1500, l'écart entre les deux branches se règle grâce à une vis horizontale. Le compas à pointe sèche, déjà utilisé pour mesurer les distances, mettre à l'échelle ou copier des dessins, se modifie avec l'apparition d'une articulation à tenon ${ }^{32}$. La copie devient encore plus aisée lorsque le compas est muni de trois branches qui permettent de transférer trois points en même temps d'un dessin à un autre. Ce compas, dit triangulaire, sert également à prendre des mesures sur des globes. Enfin, le compas sphérique, ou de calibre, mesure des formes cylindriques ou sphériques, notamment les boulets de canon $^{33}$. Les règles, instruments de mesure par excellence, sont également modifiées.

Les hommes de la Renaissance tentent de trouver des équivalents à des échelles locales dont les valeurs sont très différentes d'un lieu à l'autre. Les dimensions anthropomorphes compliquent les correspondances entre les systèmes. Dès 1500 , on fabrique des règles à échelle en bronze aux mesures linéaires. Le but est de parvenir à une échelle aux proportions constantes utilisable en tout lieu. L'introduction des chiffres arabes facilite la mise en place des échelles déterminées. Les échelles en pied persistent, mais les règles indiquent à la fin du XvI $I^{\mathrm{e}}$ siècle les divisions de villes et régions différentes. Des passerelles se mettent en place afin de faciliter le passage de mesures d'un lieu à l'autre ${ }^{34}$. Les règles commencent à être faites de laiton, plutôt que de bois, et elles sont souvent pliables, ce qui facilite leur transport. La copie préoccupe les techniciens car, avec les nouvelles possibilités de mise à l'échelle, elle autorise à modifier la taille des dessins initiaux, notamment à passer d'une maquette à la grandeur nature.

L'architecte Palladio (1508-1580) utilise des piquoirs, instruments à pointe d'aiguilles. Les traces laissées par les pointes permettent de repérer un angle ou de tracer les contours du dessin sur un morceau de papier semblable placé en dessous. Les peintres les utilisent pour reporter les dessins sur un support puis ils repassent sur les trous ${ }^{35}$. Le report d'un dessin nécessite de faciliter son agrandissement ou sa réduction ${ }^{36}$. Léonard de Vinci dessine vers 1495 un compas de réduction fixe à pointes sèches et un autre à branches fendues ${ }^{37}$. De même, Daniel Speckle (1536-1589), 
ingénieur militaire allemand, représente six compas fixes dans son Architectura ${ }^{38}$. Jost Bürgi (1522-1632), horloger et fabricant d'instruments suisses, intègre les échelles géométriques et trigonométriques aux compas. Bion insère dans son livre une version fixe et une version mobile ${ }^{39}$.

L'élargissement de l'horizon intellectuel amène un besoin de précision chez les praticiens. Les fabricants allemands profitent d'un artisanat en plein développement et d'une position géogra- phique centrale en Europe. Les élites européennes voient d'un bon œil ces avancées techniques qui accompagnent les précisions des savoirs géographiques, mathématiques ou artistiques. Les premiers états de l'art publiés peuvent être en partie imaginaires et on ne sait pas si les instruments présentés sont utilisés dans la majorité des ateliers. Toutefois, les milieux des fabricants, des élites et des savants sont déjà contaminés par un souci d'exactitude.

\section{Le Grand Siècle des instruments : fascination et rationalisation}

Durant le $\mathrm{xvII}^{\mathrm{e}}$ siècle, le nombre d'échoppes augmente dans toute l'Europe. Les élites européennes continuent de se passionner pour les instruments et de nouvelles demandes émanent des topographes, ingénieurs et navigateurs.

\section{L'affirmation de Londres et Paris}

Les fabricants d'instruments alors installés dans le centre de Londres débutent un déplacement vers l'ouest de la ville. Après un apprentissage dans un atelier près de St Clement Danes Church, Elias Allen (1588-1653) devient maître de la corporation des horlogers. En 1616, il fabrique et vend des cadrans solaires et des instruments scientifiques sur le Strand ${ }^{40}$. Son apprenti, Ralph Greatorex (1625-1712), est mentionné dans le journal de Samuel Pepys lorsqu'il évoque ses recherches d'instruments pour l'Ami- rautét1. Néanmoins, on rencontre aussi des fabricants, brodeurs, tailleurs ou épiciers qui ont tous en commun la maîtrise des poids et des mesures ${ }^{42}$. Dès 1660 , la Royal Society discute et échange des procédés nouveaux. Si l'institution est un instrument du pouvoir, elle admet en son sein les principaux fabricants. Savants et inventeurs s'y rencontrent. À Paris, les échoppes se situent autour du quai de l'Horloge. Daniel Chorez invente un télescope (1612), un binocle, des lunettes de précision (1680) et fabrique des compas de proportion. Jusque dans les années 1650, les fabricants peuvent être membres de la corporation des couteliers mais celle des fondeurs tend à devenir la référence. Le métier est peu à peu réglementé pour mettre en place des critères et normes de qualité. Pierre Sevin (actif entre 1662 et 1685), membre de la corporation des fondeurs, produit des graphomètres précis. Il fréquente l'académicien 
Joseph Sauveur mais ne fait pas officiellement partie de l'institution ${ }^{43}$. Enfin, $\mathrm{d}$ 'origine anglaise, Michael Butterfield (1635-1724) s'installe au Faubourg SaintGermain en 1663 et devient rapidement ingénieur du roi. Proche de l'Académie, il travaille aux niveaux du château de Versailles. Butterfield réalise des équerres pliables, des règles à échelles et des rapporteurs signés "Butterfield Paris $^{44}$ ». Au xviI ${ }^{\mathrm{e}}$ siècle, la fabrication est marquée par la conception de boîtes $d$ 'instruments très complètes et $d$ 'instruments luxueux destinés aux élites.

\section{Les boîtes à instruments : éloge à la technique}

Les fabricants fournissent des boîtes de grande qualité aux collectionneurs dès le début du $\mathrm{xvII}^{\mathrm{e}}$ siècle. La fascination pour le tracé exact est à son apogée : compas triangulaire, elliptique, d'ellipse, de réduction ou de navigation s'invitent dans ces boîtes (figure 2) ${ }^{45}$. L'Anglais Roger North (1651-1734), architecte amateur, acquiert un nécessaire complet d'instruments mathématiques ${ }^{46}$. La boîte de North, fabriquée sur mesure vers 1680 , se divise en quatre compartiments ${ }^{47}$. Le premier contient des compas et leur encre. Le deuxième tiroir accueille un porte-crayon, un tire-ligne, une ardoise, une règle coulissante en buis

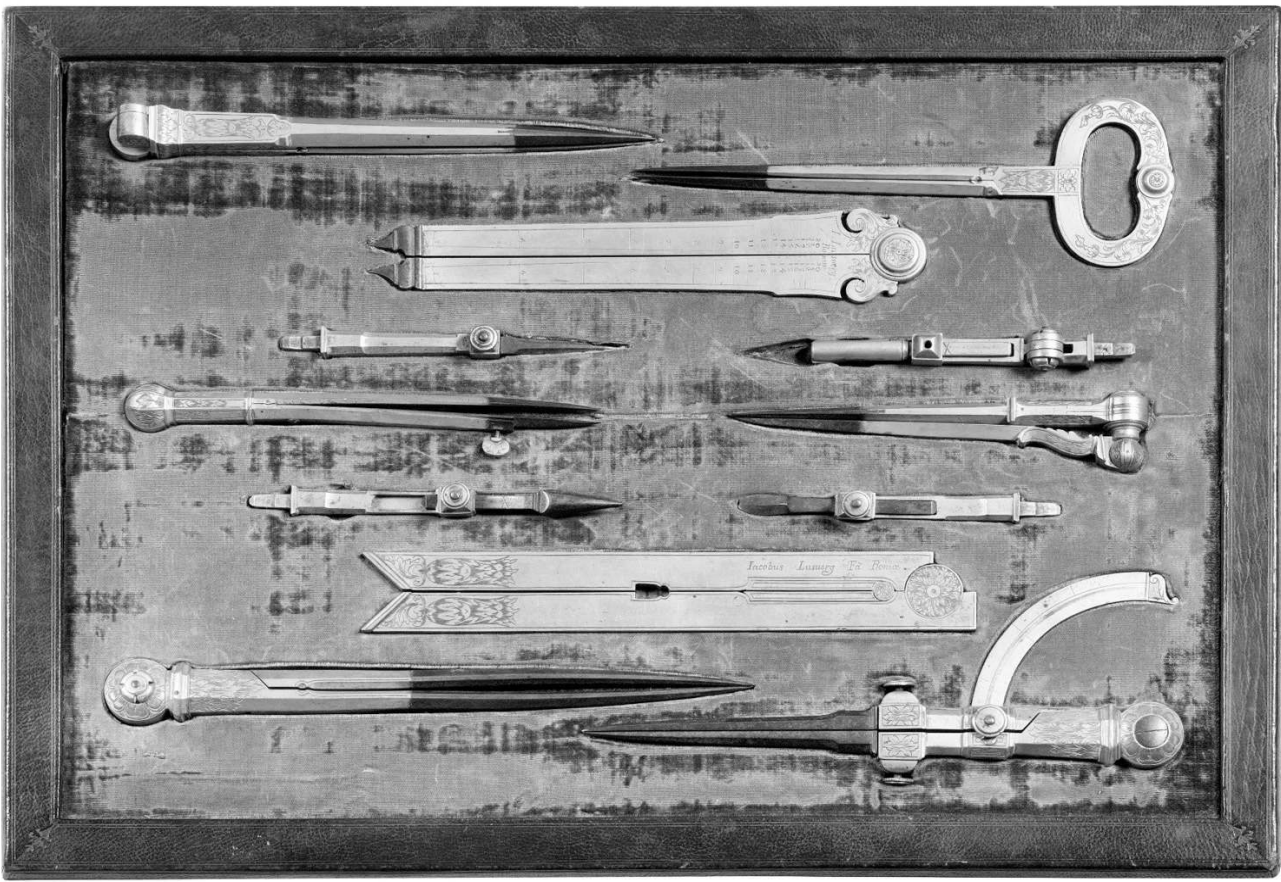

Fig. 2. - Instruments de dessin dans leur boîte, 1683. Ensemble de huit instruments à dessiner en laiton doré et acier. Signé Iacobus Lusuerg et daté 1683. Ancienne collection Jacques Doucet, aujourd'hui dans une collection particulière. 
et une règle de calcul. Des accessoires pour le grand compas et un segment de courbe de dessin se situent dans le troisième compartiment. Enfin, le quatrième tiroir contient un cadran gravé à chiffres romains, une équerre de dessin triangulaire $60^{\circ}$, de la ficelle, un fil à plomb, une boîte à poncif et un cadran universel. Ces instruments sont-ils véritablement destinés au travail quotidien du dessin? Non. La majorité des outils véritablement utilisés a disparu. Ces boîtes sont des mises en perspective du savoir. Précieuses, elles se regardent, s'admirent et fascinent. Néanmoins, le propriétaire peut aussi les toucher et utiliser les instruments pour son loisir. Cet amour de l'art de la précision se matérialise dans les collections. La boîte est un spectacle de l'ingéniosité des artisans, du travail et de l'art de la technique. Elle est un éloge au savoir-dessiner dont les outils continuent de se modifier.

\section{"Afin de mesurer à un cheveu près"}

L'encre de carbone est désormais utilisée couramment. L'anneau coulissant du tire-ligne devient inutile car la composition du métal permet une ouverture fixe et une ligne constante. Une des innovations du siècle réside dans la plume technique. En 1636, le mathématicien allemand Daniel Schwenter décrit un modèle primitif de plume à réservoir et portemine muni d'un ressort ${ }^{48}$. Vers 1659, Hans Baumann, charpentier de Nuremberg, fabrique et vend des crayons graphite. Son emploi se généralise grâce à l'exploitation d'une mine au Borrowdale en 1664. John Pettus décrit en 1683 un dry pencil : un gra- phite entouré d'une gaine de sapin ou de cèdre ${ }^{49}$. La maniabilité est considérablement améliorée, mais peu de praticiens l'utilisent avant le XIX ${ }^{\mathrm{e}}$ siècle. Des nouvelles améliorations concernent également les compas. Jacob Leupold propose un large éventail de compas comprenant notamment des compas à balustre, à roulette ou à pointillés ${ }^{50}$. Le compas «à cheveux » est caractéristique des ambitions $\mathrm{du} \mathrm{XvII}^{\mathrm{e}}$ siècle. La pointe est maintenue par un ressort et le réglage de l'ouverture à partir d'une petite vis permet « de mesurer à un cheveu près ». Représenté dès 1728 par Chambers (1680-1740), le compas à cheveux est sans doute utilisé dès le début du siècle ${ }^{51}$. Quant au compas à balustre, il autorise le dessin de petits cercles en 1700. Des versions « de poche » existent pour être insérées dans des boîtes d'instruments portatives. Les dessinateurs sont toujours préoccupés par la reproduction. Le compas triangulaire s'invite dans les encyclopédies d'instruments. La lecture des cartes est facilitée $e^{52}$. Le piquoir est modifié pour faciliter sa prise en main et améliorer sa précision. Joseph Moxon représente un " protracting pin $^{53}$ ». Réalisé en laiton, il s'agit d'un support « deux en un » qui accueille un tire-ligne ou une aiguille, et dont les mines sont protégées par un capuchon. En 1605, le mathématicien et astronome jésuite, Christophe Scheiner (1575-1650), est reçu à la cour du duc Guillaume V à Munich. Il lui explique le fonctionnement du pantographe qu'il a inventé deux ans auparavant. En 1631, Scheiner décrit sa fabrication et son usage dans un ouvrage. L'invention fascine. Samuel Pepys (1633-1703), haut fonctionnaire de l'Amirauté anglaise, membre du Parlement et diariste anglais, 
se réjouit de cette nouveauté. Le 13 janvier 1668, après avoir visité le fabricant d'instruments londonien Henry Wynne, il écrit :

" J'ai eu très grand plaisir, non pas à cause de son ingéniosité en général, mais en particulier, lorsqu'il me fit une démonstration de l'usage du parallélogramme, grâce auquel il dessina en un quart d'heure sous mes yeux, en petit, une carte nette $\mathrm{d}^{\prime}$ Angleterre à partir d'une grande [...]. Il me le faut, et par conséquent, je désire commander un exemplaire de ce dont je viens de parler ${ }^{54}$.»

Toutefois, les instruments ne sont pas adoptés par tous les dessinateurs. Les habitudes comptent. Des dessins en soierie de César Galais (1811-1891) sont intégralement piqués afin de les reproduire sur un second papier. À la fin du
xIX $x^{e}$ siècle, à Sèvres, Donzé utilise encore la technique du piquage pour reporter des décorations d'assiettes ${ }^{55}$. Le temps de l'invention ne correspond pas obligatoirement au temps de l'utilisation dans le cadre de l'atelier.

Londres et Paris sont devenus au $\mathrm{XVII}^{\mathrm{e}}$ siècle les centres européens de la fabrication des instruments. Ce dynamisme stimule les artisans et les praticiens. L'intérêt des publics est de plus en plus grand. Posséder une boîte à instruments montre son appartenance à une classe privilégiée mais aussi son goût pour les innovations et les techniques. Les hommes du XvII ${ }^{\mathrm{e}}$ siècle sont fascinés par les beaux objets et la boîte devient un cabinet de curiosité. Parallèlement, de nombreuses améliorations sont réalisées. On retiendra la justesse, la maniabilité des instruments et la mise en place de mécanismes plus complexes.

\section{Des gammes d'instruments pour un langage universel (xvIII siècle)}

Durant le xvIII ${ }^{\mathrm{e}}$ siècle, différentes catégories professionnelles ont besoin d'outils fiables, les ingénieurs civils et militaires, les géomètres, les couturières, les modistes, les dessinateurs de fabrique, les orfèvres... et les collectionneurs riches et cultivés sont toujours aussi intéressés. L'instrument perfectionné forme l'alphabet d'un langage universel. Il est pensé comme un outil de communication.

\section{Fabricant-entrepreneur?}

En France, des dynasties de fabricants, comme les Chapotot, les Sevin, tiennent des échoppes. Parmi eux, Claude Langlois fonde un atelier de fabrication vers 1730 . Installé dans une galerie du Louvre, il conçoit un pantographe, des instruments de mathématiques et des règles ${ }^{56}$. Langlois fournit l'Observatoire de Paris. Son neveu, Canivet, perfectionne des règles, des équerres et un pantographe salué par l'Académie royale 
des sciences en $1743^{57}$. Aucun fabricant n'est membre de l'Académie des sciences. Parallèlement, les fabricants londoniens cumulent la publication de traités, la fabrication et l'exportation d'articles. Edward Scarlett (1688-1743), opticien de George II, édite une carte commerciale caractéristique de l'appli- cation concrète des sciences et des techniques au quotidien et de la recherche de confort typique des Lumières (figure 3$)^{58}$. Publicité précoce, datée de 1714-1727, elle insiste sur l'innovation principale de l'opticien : des lunettes munies de branches reposant sur les oreilles grâce à une forme en spirale. Elles fournissent une stabi-

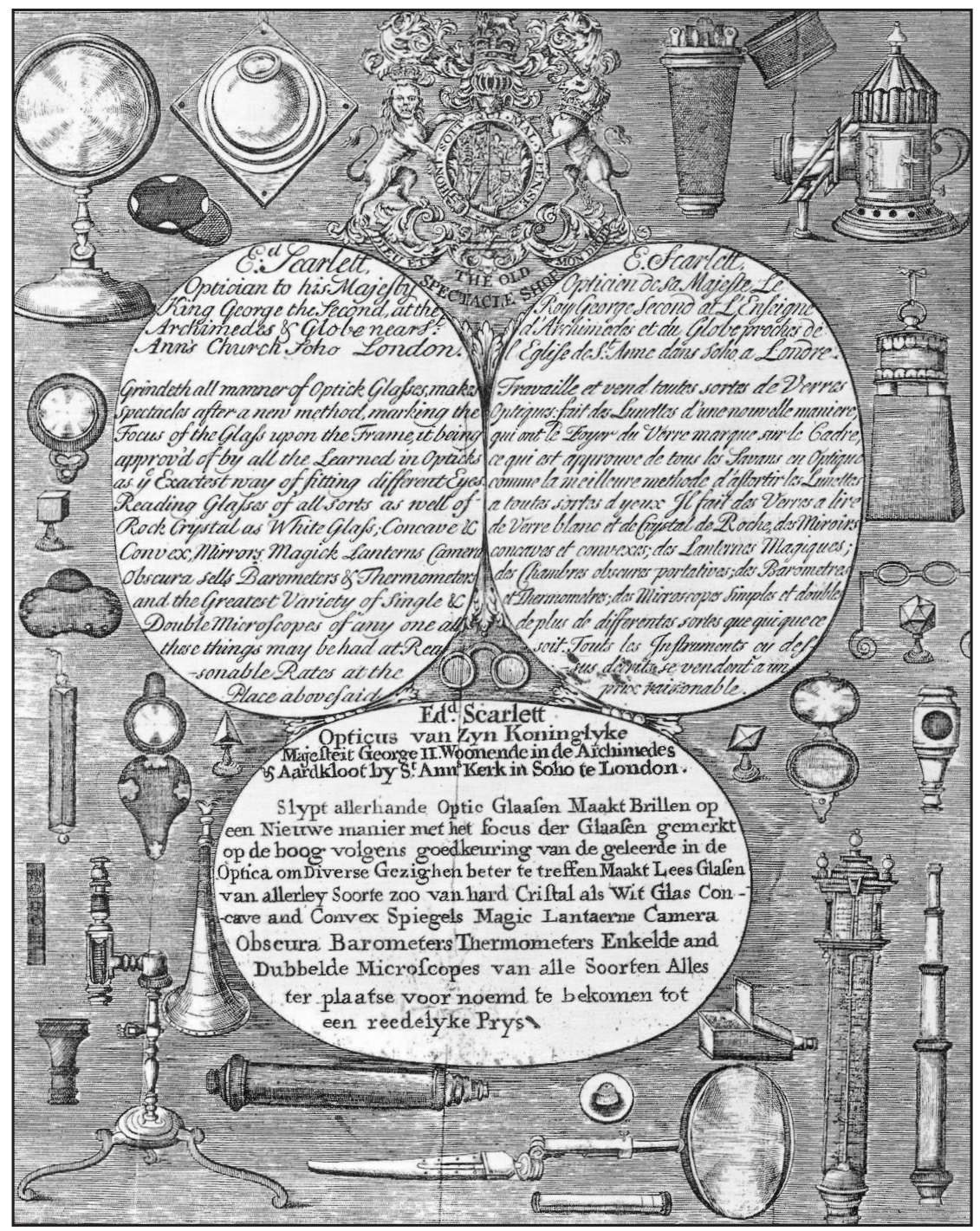

FIG. 3. - Carte commerciale de l'opticien Edward Scarlett. Bodleian Library, University of Oxford, Douce Adds 139 (766). 
lité considérable au porteur. Le même texte, en anglais, français et néerlandais, est inséré dans trois formes sphériques. La carte témoigne du développement de la profession d'opticien tournée vers les innovations techniques. Ces lunettes sont à vendre en boutique lors des jours ouvrables et donc relativement accessibles $^{59}$. Thomas Heath (actif entre 1720 et 1772) entre dans la corporation des épiciers et installe sa boutique d'instruments sous le nom "The Hercules \& Globe in the Strand ${ }^{60}$ ». Sans suivre d'apprentissage commercial, Benjamin Martin s'installe en autodidacte au 171 Fleet Street en 1755. Il réalise des conférences scientifiques, publie une trentaine d'ouvrages sur les instruments et perfectionne un pantographe. Il est un véritable entrepreneur. Si les fabricants se comportent à la fois comme des praticiens et des entrepreneurs dont le but est de proposer de nouveaux produits aux clients, ils ne sont pas pour autant en nombre croissant dans la capitale.

\section{Une offre plus importante mais un nombre de fabricants stable (Paris, 1600-1789)}

Une analyse du Minutier central des notaires parisiens permet de se faire une idée du petit nombre de fabricants présents dans la capitale. Néanmoins, avant 1610, aucune mention de fabricants dans un contrat de mariage, d'apprentissage ou autre minute notariale n'a été retrouvée. Entre 1611 et 1650, trois fabricants d'instruments de mathématiques sont recensés. L'un d'entre eux est dit maître horloger ${ }^{61}$. Durant les cinquante années qui suivent, cinq fabricants ont laissé une trace dans le minutier ${ }^{62}$. Quatre fabricants différents sont recensés entre 1701 et $1750^{63}$. Les fabricants d'instruments de mathématiques sont à peine plus nombreux, cinq, jusqu'à la Révolution ${ }^{64}$. En dehors d'une croissance après 1600, les fabricants ne semblent pas nombreux à Paris. Bien entendu, la source utilisée ne permet de voir qu'une partie de l'iceberg. Toutefois, le métier est très spécialisé et les praticiens ne désirent pas changer des manières de faire qui donnent de bons résultats. De plus, les prix sont sans doute assez élevé pour que la demande se modifie du tout au tout. La pratique quotidienne du métier permet aussi aux exécutants de repousser les limites des possibles avec leurs propres outils. Un des fils directeurs du XVIII $^{\mathrm{e}}$ siècle réside dans la volonté de stabiliser des normes afin d'universaliser les savoirs. Les outils et instruments sont un des moyens, parmi d'autres, d'y parvenir.

\section{Pour des normes universelles}

Vers la fin du siècle, le tire-ligne est désormais appelé " plume à dessiner ». L'ajout de laque dans l'encre de carbone fournit aux techniciens une encre indélébile. À cette nouveauté s'ajoutent un système d'alimentation au comptegouttes et une lame pivotante pour faciliter le nettoyage ${ }^{65}$. La plume à dessiner est l'ancêtre du porte-plume. Un piquoir et un crayon sont souvent intégrés à la partie supérieure. En 1761, à Stein, près de Nuremberg, l'ébéniste Kapsar Faber fonde la manufacture Faber Castel dont le succès est encore d'actualité. Des tiges graphites insérées dans des gaines de bois sont réalisées en série ${ }^{66}$. Parallèlement, 
Edward Naine (1726-1806), fabricant d'instruments, utilise le caoutchouc, alors réservé aux semelles, pour effacer autour de 1788. Chambers en 1728 et Bion en 1758 représentent un compas aux extrémités réversibles; l'instrument permet d'utiliser soit deux pointes sèches, deux mines ou une pointe sèche et une mine ${ }^{67}$. Néanmoins, il manque de fiabilité : le compas fait des écarts incontrôlables pendant le tracé. La règle à échelle connaît peu de changements mais, à la fin du siècle, celle de Brander \& Hoeschel comporte les échelles en pouces de six villes différentes ${ }^{68}$. De plus, l'inscription sur les règles $\mathrm{d}^{\prime}$ une division décimale en pouce est plus courante. La

\section{Conclusion}

Les modifications des instruments de dessin sont caractéristiques des changements intellectuels, économiques et sociaux de la Renaissance aux Lumières. Durant les $\mathrm{Xv}^{\mathrm{e}}$ et $\mathrm{XvI}^{\mathrm{e}}$ siècles, les explorations maritimes et les développements artistiques entraînent une réflexion sur la manière de représenter le monde. Grâce au développement de l'imprimerie, les publications offrant un état de l'art des instruments se multiplient. Des centres tels que Nuremberg se retrouvent sur le devant de la scène, notamment grâce aux nouveaux secteurs de l'artisanat (horlogerie) et à la présence de minerai nécessaire à la fabrication d'instruments. Néanmoins, si les Grands perçoivent bien l'intérêt pour leur rayonnement dans la maîtrise des sciences et des techniques, ce sont les artisans-fabricants qui, au jour le jour, interrogent leur propre pratique. facilité d'un passage d'une mesure locale à l'autre et la mise en place d'une mesure universelle caractérisent les Lumières. Enfin, en 1794, le système métrique est introduit pour remplacer le " pied du roi ». Néanmoins, l'ancien système, " pied de France » est encore utilisé ${ }^{69}$.

Le fabricant d'instruments du siècle des Lumières a plusieurs cordes à son arc. Il publie ses résultats, pratique et interroge les limites des techniques. Le fabricant est devenu un entrepreneur et il produit désormais en série. Toutefois, le mot d'ordre du siècle est l'universalité des instruments. Ils doivent être utilisables et compris par tous pour mettre en place un langage stable.

Durant le $\mathrm{xvII}^{\mathrm{e}}$ siècle, la reconnaissance des scientifiques via des institutions comme les académies joue un rôle de diffuseur auprès des publics. Si les fabricants français ne sont pas intégrés aux nouvelles institutions, cela n'empêche pas certains d'avoir des liens étroits avec leurs membres. En Angleterre, la technique bénéficie d'une plus haute estime. De même, Colbert met en ordre les corporations et les métiers correspondant à chacune. Outre-Manche, le système semble plus souple. Toutefois, des deux côtés, des amateurs toujours plus nombreux stimulent la production d'instruments de luxe. Le siècle suivant est marqué par la volonté de communiquer dans toutes les situations. En résumé, les instruments sont transportables, pliables et régulièrement interrogés pour être améliorés par les praticiens et 
savants. Le siècle de l'Encyclopédie tente de mettre en place des savoirs universels, en stabilisant les mesures, et de les rendre accessibles par la mise en série. Tout au long de la période, de nombreux acteurs sont concernés : les pouvoirs au plus haut niveau de l'État, les praticiens et les savants. Néanmoins, ce n'est pas encore au $\mathrm{XVIII}^{\mathrm{e}}$ siècle que les praticiens adoptent des nouveautés qui peuvent largement modifier leurs habitudes. À l'aube du xix $x^{\mathrm{e}}$ siècle, les instruments de dessin deviennent les outils d'un langage commun soutenant ainsi la diffusion des savoirs, gommant les frontières et outils de la globalisation.

\section{Notes}

1. Léonard DE VINCI, Le compas de proportion pour les rapports de 1/2, 1/4, et $1 / 8$ comme expérience pour les transformations des figures, Victoria and Albert Museum, Londres, "Codex Forste ", 3 vol., F. 141, et Institut de France, manuscrits B, F, H et L. Robert Latham et William Mattews, The Diary of Samuel Pepys : A New and Complete Transcription, Berkeley, University of California Press, vol. 10, p. 392.

2. Monique Pelletier, Les cartes des Cassini, la science au service de l'État et des provinces, Paris, CTHS, 2013; Christian Grataloup, Représenter le monde, Paris, La documentation française, $\mathrm{n}^{\circ}$ 8084, 2011; Jean-Jacques Levallois, Mesurer la Terre, Paris, AFT, 1988.

3. Erwin PANOFSKY, La perspective comme forme symbolique (1932), trad. fra. Paris, éd. de Minuit, 1975; Yves Pauwels, « Discours graphique et discours du texte dans les traités d'architecture français du XVI ${ }^{e}$ siècle ", Humanistica, An International Journal of Early Renaissance Studies, 2013, vol. VII, p. 235-238. Consulter notamment la base Architectura du Centre d'études supérieures de la Renaissance de l'université François Rabelais (Tours).

4. Maurice Daumas, Les instruments scientifiques aux XVII' siècle et XVIII' siècle, Paris, PUF, 1953; Bertrand Gille (dir.), Histoire des techniques, Paris, Gallimard, 1978.

5. Richard SennetT, Ce que sait la main. La culture de l'artisanat, trad. fra. Paris, Albin Michel, 2010; Matthew B. CRAWFord, Éloge du carburateur. Essai sur le sens et la valeur du travail, rééd. Paris, La Découverte, 2016.
6. Gianenrico Bernasconi, Objets portatifs au Siècle des Lumières, Paris, CTHS, 2015; Liliane Hilaire-PÉrez, La pièce et le geste : artisans, marchands et savoir technique à Londres au XVIII' siècle, Paris, Albin Michel, 2013; Pascal DubourGGlatigny, "Mécaniser la perspective : les instruments entre pratique et spéculation ", e-phaïstos, 2013, vol. II, $\mathrm{n}^{\circ}$ 1, p. 23-33; id., Il disegno naturale del mondo : saggio sulla biografia di Egnatio Danti, con edizione del carteggio, Pérouse, Aguaplano, 2011; Pascal Dubourg-Glatigny, Hélène Vérin (dir.), Réduire en art, la technologie de la Renaissance aux Lumières, Paris, Presses de la Maison des sciences de l'homme, 2008; Hélène VéRIN, La gloire des ingénieurs. L'intelligence technique $d u \mathrm{XVI}^{e}$ au XVIII siècle, Paris, Albin Michel, 1993.

7. Alexandre Koyré, « Du monde de 1'“à-peuprès" à l'univers de la précision », Études d'histoire de la pensée philosophique, Gallimard, 1977, p. 341362.

8. Peter Burke, La Renaissance européenne, Paris, Le Seuil, 2000.

9. Pour un bref aperçu : Stillman DraKe et Israel E. Drabkin, Mechanics in Sixteenth-Century Italy. Selections from Tartaglia, Benedetti, Guido Ubaldo \& Galileo, Madison, Wisconsin, 1969; Paul Ive, The Practise of Fortification (1589). English Experience, Londres, Da Capo Press, 1968; Rodney W. SHIRLEY, The Mapping of the World. Early Printed World Maps 1472-1700, London, New Holland Press, 1993; Léon VoET, The Golden Compasses. A History and Evaluation of the Printing and Publishing Activities of the Officina Plantiniana at Antwerp, London, Routledge, 19691972.

10. P. Dubourg-Glatigny, H. Vérin (dir.), Réduire en art..., op. cit.; E. PANOFSKY, La perspective..., op. cit.

11. Filippo Brunelleschi (1377-144) explore à Florence les formes architecturales romaines. Leur compréhension et leur application nécessitent la réalisation de dessins à grande échelle. Les instruments de dessin, règle large et plate équerre, compas à pointe sèche et compas ordinaire, revêtent une importance capitale pour le métier. Eugenio BAtтisti, Brunelleschi : the complete work, London, Thames and Hudson, 1981.

12. En mathématiques, la projection orthogonale est une transformation de l'espace via une application linéaire. En géométrie plane, c'est une projection où les deux droites sont perpendiculaires. En géométrie dans l'espace, c'est une projection où la droite et le plan sont perpendiculaires. Le traité de Vitruve, De Architectura, est une référence pour les techniques de construction de l'Antiquité classique.

13. Michael Twyman, L'imprimerie : histoire et techniques, Lyon, ENS/Institut d'histoire du livre, 2007. 
14. H. W. Dickinson, "A brief history of draughtsman's instruments ", Transaction of the Newcommen Society, 1949-1950, vol. 27, p. 73-84.

15. René DE Lespinasse et François BonNARDOT, Les métiers et corporations de la Ville de Paris, Le Livre des Métiers d'Étienne Boileau, Paris, Imprimerie nationale, 1879, p. 40; Maurice Daumas, "Quelques fabricants d'instruments scientifiques anciens ", Revue d'histoire des sciences et de leurs applications, 1950 , vol. 3, n ${ }^{\circ}$ 4, p. 364-370.

16. Augustine Ryther, 52 cartes à jouer, $9,5 \times 5,7 \mathrm{~cm}$, 1590, British Museum, 1938,0709.57.1-60; Elizabeth BAIgENT, «Ryther, Augustine », Oxford Dictionary of National Biography (online ed.), Oxford University Press, 2014 [http://dx.doi.org/10.1093/ref : odnb/ 24428].

17. Philippe Danfrie, Déclaration de l'usage $d u$ graphomètre, par la pratique..., Paris, Chez ledict Danfrie, 1597; M. Daumas, Les instruments..., op. cit., p. 24-25.

18. Philippe Renouard, Imprimeurs parisiens, libraires, fondeurs de caractères et correcteurs d'imprimerie, depuis l'introduction de l'imprimerie à Paris (1470) jusqu'à la fin du XVI siècle : leurs adresses, marques, enseignes, dates d'exercice, notes sur leurs familles, leurs alliances et leur descendance, d'après les renseignements biographiques et les documents inédits, Paris, A. Claudin, 1898, p. 91.

19. Richard Sennett a proposé un examen du travail artisanal au quotidien. R. SEnNett, Ce que sait..., op. cit., 2010.

20. Henri MicheL, Catalogue des instruments connus d'Érasme Habermel, Nationaal Centrum voor de Geschiedenis van Wetenschappen, Centre national d'histoire des Sciences, manuscrit, 1937.

21. L'Observatoire de Paris conserve quinze " instruments universels ». W. ЕскHARDT, " Erasmus Habermel : Zur Biographie des Instrumentenmachers Kaiser Rudolfs II », Jahrbuch der Hamburger Kunstsammlungen, 1976, $\mathrm{n}^{\circ} 21$, p. 55-92.

22. Lucia NuTI, Ritratti di città. Visione e memoria tra Medievo e Settecento, Venice, Marsilio, 1996.

23. B. GILle (dir.), Histoire des techniques, op. cit.; Alex KelLER, «The missing years of Jacques Besson, inventor of machines, teacher of mathematics, distiller of oils, and Huguenot pastor ", Technology and Culture, 1973, n 14, p. 28-39; François Russo, « Deux ingénieurs de la Renaissance - Besson et Ramelli ", Thalés, 1948, n 5, p. 108-112.

24. Benjamin RAVIER, Voir et concevoir : les théâtres de machines (XVI-XVIII siècle), thèse de doctorat, Université Paris 1, 2013.

25. Siegmund GÜNTHER, "Lencker, Hans », Allgemeine Deutsche Biographie (ADB), Leipzig, Duncker \& Humblot, 1883, notice 249.
26. Monique Zerdoun Bat-Yehouda, Les encres noires au Moyen Âge : jusqu'à 1600, Paris, CNRS, 1983.

27. Nicolas Bion, Traité de la construction et principaux usages des instruments de mathématique, Paris, Chez Jean Boudot, 1709, pl. 9, p. 94.

28. Alfred Wainwright, A Pictorial Guide to the Lakeland Fells, Western Fells, London, Frances Lincoln, 2005, livre 7; Conrad Gesner, De omni rerum fossilium genere, gemmis, lapidibus, metallis, et huiusmodi, libri aliquot, plerique nunc primum editi, Zurich, Naturforschende Gesellschaft, 1565.

29. Il est déjà utilisé chez les Égyptiens et les Romains.

30. Compas à brisures interchangeables ou pointes porte-crayon, Salon de Dresde, v. 1550. Ce salon n'est jamais abandonné. En 1746, la collection prend le nom de Mathematisch-Physikalischer Salon. Devenu musée, c'est à partir de ces instruments qu'est établi le temps officiel, le "Greenwich » de la Saxe. Chisholm Hugh, " Albert III (duke of Saxony) ", Encycloprdia Britannica, Cambridge, Cambridge University Press, 1911.

31. Giovanni Pomodoro, Geometrica prattica, Rome, S. Paolini, 1599; N. Bion, Traité de la construction, op. cit., pl. 8.

32. Boîte à dessin dite "Bartholomew Newsum ", British Museum, Londres, 1570.

33. N. Bion, Traité de la construction, op. cit., p. 86.

34. Boîte à dessin milanaise contenant une règle pliante graduée, Museum of the history of science, Oxford, début $\mathrm{xVI}^{\mathrm{e}}$ siècle. De conception allemande, boîte contenant des instruments de géomètreingénieur militaire, avec des règles en laiton dorées et argentées, Museo di Storia della Scienza, Florence, v. 1599.

35. Ensemble milanais, British Museum, Londres, 1570 .

36. Héron d'Alexandrie, mathématicien, du $\mathrm{I}^{\text {er }}$ siècle, a travaillé sur le calcul des dimensions pour la transposition des dessins. Philippe FleurY, "Vitruve et Héron d'Alexandrie. À propos des techniques dites "Pneumatiques" ", dans Gilbert Argoud, Sciences et vie intellectuelle à Alexandrie ( $I^{e r}-I I I^{e}$ siècle après J.-C.), Saint-Étienne, Presses de 1'Université de Saint-Étienne, 1994, p. 67-81. Un compas de réduction a été retrouvé à Pompéi, 79 ap. J.-C. (reproduction au Science Museum de Londres).

37. Léonard de Vinci, dessin de ses carnets, v. 1493, Paris, Institut de France, manuscrit H, $1493-4$

38. Daniel Speckle, Architectura von Vestungen. Wie die zu vnsern zeiten mögen erbawen werden, an Stätten, Schlössern unn Clussen, zu Wasser, Land, Berg unn Thal..., Strasbourg, Bernhart Jobin, 1589. 
39. Ludwig OechsLIN, Der Bürgi-Globus : Technik und Kultur, Zurich, Schweizerisches Landesmuseum, 2000.

40. Gerard L'Estrange Turner, Elizabethan Instrument Makers : The Origins of the London Trade in Precision Instrument Making, Oxford, Oxford University Press, 2000, p. 31-32.

41. Arthur Bryant, Samuel Pepys : The Saviour of the Navy, Londres, The Reprint Society, 1943, p. 140.

42. Joyce Brown, Mathematical InstrumentMakers in the Grocers' Company (1688-1800), London, Science Museum, 1979, p. 77.

43. Stéphane VAn Damme, Paris, capitale philosophique : De la Fronde à la Révolution, Paris, Odile Jacob, 2005, p. 131.

44. Ibid., p. 131-134.

45. Au même moment le pantographe se répand. Il offre une solution mécanique aux problèmes liés à la reproduction, la copie, l'agrandissement et la réduction des dessins. La facilité de reproduction devient une recherche centrale durant le XVIII ${ }^{\mathrm{e}}$ siècle.

46. Hugh Chisholm, "North, Roger ", Encyclopædia Britannica, Cambridge, Cambridge University Press, 1911, vol. 19, p. 759.

47. La boîte de North est conservée au Jesus College à Cambridge.

48. Daniel Schwenter, Deliciae physico-mathematicae, Nuremberg, Verlegung Jeremiae Dümlers, 1636. La planche 9 de Bion représente aussi une plume technique (op. cit.).

49. Eric VoICE, " History of the Manufacture of Pencils ", Transactions of the Newcomen Society, 1950, $\mathrm{n}^{\circ}$ 27; John Petrus, Fleta Minor, or the Laws of Art and Nature..., London, Thomas Dawks, 1683. Cet ouvrage est une traduction de Aula Subterranea, de Lazarus Ercker, paru à Prague en 1574.

50. Jacob Leupold, Theatrum arthmetico-geometricum, Leipzig, gedruckt bey Christoph Zunkel, 1727.

51. Ephraim Chambers, Cyclopædia : or An Universal Dictionary of Arts and Sciences, London, J. Knapton, 1728, vol. 1.

52. Bion en représente un compas triangulaire dans son ouvrage de 1709. Un bel exemple daté de la fin du siècle est conservé au Germanisches Nationalmuseum de Nuremberg.

53. Jospeh Moxon, Mathematicks Made Easie, Or A Compleat Mathematical Dictionary, London, W. Hawes, 1701.

54. R. Latham et W. Mattews, The Diary..., op. cit., p. 392.

55. Musée des Arts décoratifs (Paris), fonds Galais, et Archives de la Manufacture de Sèvres, coll. graphique, travaux d'assiettes, Donzé, 1880.
56. M. Daumas, « Quelques fabricants d'instruments scientifiques anciens ", op. cit., p. 364370 .

57.S. VAN DAMme, Paris, capitale..., op. cit., p. 131; François-Joseph-Michel NoËL et M. CARPENTIER, Nouveau dictionnaire des origines, inventions et découvertes dans les arts..., Paris, Janet et Cotelle, 1840, p. 373; Claude LANGLoIs, Description et usage du pantographe, autrement appelé singe : changé $\mathcal{E}$ perfectionné par C. Langlois, ingénieur du Roi $\mathcal{E}$ de l'Académie royale des sciences pour les instrumens de mathématiques, s. 1., 1744.

58. Christoph Heyl, A Passion for Privacy. Untersuchungen zur Genese der bürgerlichen Privatsphäre in London, 1660-1800, Munich, Oldenbourg, 2004.

59. G. L'Estrange Turner, Nineteenth-Century..., op. cit., 1983.

60. Lewis Pyenson, Jean-François Gauvin, L'art d'enseigner la physique : les appareils de démonstration de Jean-Antoine Nollet (1700-1770), Sillery, Septentrion, 2002.

61. AN, MC/ET/CV/783, Charbonnier Philippe, fabricant d'instruments de mathématiques, demeurant à la Porte Saint-Jacques: accord entre Nicolas Milochin l'aîné, mégissier à Paris, rue de Lourcines, et Philippe Charbonnier comme acquéreur d'une maison sise Grande rue Mouffetard, enseigne des Trois rois, 31 mai 1641. MC/ET/III/556, Blondeau Baptiste, fabricant d'instruments de mathématiques demeurant Ile du Palais: mariage de Louis de Bourlon, marchand à Pontoise, en présence de Baptiste Blondeau, neveu à cause de Marie Cossart, sa femme [et de Madeleine Parison dite de Laulnay, en présence de Marie Savaria, femme de Charles Chamoys, architecte du Roi, amie de la future épouse], 23 février 1635. MC/ ET/CXXII/1571, fol. XLVIII, Avelot Mathieu, horloger au Greffe, fabricant les instruments mathématiques de la Marine, demeurant au coin de la rue Dauphine, du côté de l'hôtel de Nevers: bail à Gabriel Goussette, marchand quincaillier joaillier, d'une portion de boutique dépendant de la maison, 19 août 1610 .

62. AN, MC/ET/XVII/463, mariage de Pierre Lemaire, fondeur et fabricant d'instruments de mathématiques, et de Marguerite Viay, fille de Louis Viay, aussi "fabricateur » d'instruments de mathématiques, 19 octobre 1698. MC/ET/ CXVII/177, mariage en présence de Jean Mouton, fabricant d'instruments de mathématiques, 3 septembre 1699. MC/ET/LXII/269, mariage en présence de Pierre Sautout, fabricant d'instruments de mathématiques, 27 novembre 1699. MC/ET/ XXIV/525, alloué d'Olivier de Rouvroy, fils de Gilles de Rouvroy, maître de danse, pour deux ans, 
chez Henri Maccard, maître fabricant d'instruments de mathématiques, 12 décembre 1700 .

63. AN, MC/ET/LXX/213, mariage de Le Roy Gilles, fondeur et fabricant d'instruments de mathématiques, quai de l'Horloge, paroisse Saint-Barthélemy, avec Marie Anne Pacot, 3 janvier 1701. MC/ET/VII/183, procuration... en présence de Jean-Baptiste Delure, ingénieur du roi et fabricant d'instruments de mathématique..., 23 septembre 1708. MC/ET/II/364, mariage entre Joseph Saugrain, libraire, quai de Gesvres, et Jeanne Thoury, fille de Jacques Thoury (décédé), fabricant d'instruments de mathématiques, quai de Gesvres, 22 décembre 1709. MC/ET/CXV/439, notoriété par Gaudron Pierre, horloger ordinaire du duc d'Orléans, place Dauphine, et Jacques Delamarre lapidaire joaillier, même adresse, relative au décès de Michel Butterfield, fabricant d'instruments de mathématique et de sa femme Pierrette Varnier; héritière leur fille unique; épouse de Pierre Faveau Desgranges joailliers, 11 juillet 1724 .

64. AN, MC/ET/CXII/704/B, contrat d'apprentissage entre Tronson, Charles et Langlois, Claude, ingénieur de l'Académie des sciences, fabricant d'instruments de mathématiques, Paris, 22 juin 1751. MC/ET/LXXXII/312, contrat d'alloué entre Baradelle, Nicolas Alexandre et Manche Nicolas, fabricant d'instruments de mathématiques, 18 juillet 1751. MC/ET/XXXVIII/463, mainlevée entre Bion Jean Baptiste Nicolas, ingénieur du roi pour les instruments de mathématiques, Paris, paroisse Saint-Nicolas-du-Chardonnet, et Ferret, marchand fabricant, 16 juillet 1761. MC/ET/ VII/336, dépôt d'acte de baptême entre Menant, Pierre Louis, maître fondeur, fabricant d'instruments de mathématiques, Paris, 10 décembre 1761. MC/ET/XXVII/367, inventaire après décès de JeanBaptiste Oudry, fondeur et faiseur d'instruments de mathématiques, époux de Jeanne Françoise Malson, marchande d'éventails, demeurant rue de la Tannerie, quartier de la place de Grève, 8 octobre 1773.

65. Monique de PAs et Françoise Flieder, " Historique et étude de la composition des encres noires manuscrites ", Studies in Conservation, 1972, vol. 17, p. 943-952.

66. M. Bailly de Merlieux et A. Jullien, Memorial revue encyclopédique des connaissances humaines, Paris, direction du mémorial encyclopédique, 1835, « arts intellectuels », p. 669.

67. E. Chambers, Cyclopædia, op. cit.; N. Bion, Traité de la construction, op. cit.

68. Alto BRACHNER, Georg Friedrich Brander, 1713-1783. Wissenschaftliche Instrumente aus seiner Werkstatt, Munich, Deutsches Museum, 1983.

69. Bureau international des poids et mesures, Le système international d'unités, Sèvres, Bureau international des poids et mesures, 2006, p. 19. 